\title{
EQUAÇÃO DE CORRELAÇÃO COM ENFASE NA LITOLOGIA PARA ESTIMATIVA DE RESISTÊNCIA À COMPRESSÃO UNIAXIAL ATRAVÉS DO USO DE PUNDIT*
}

\author{
Nei Bongiorni Alff de Freitas ${ }^{1}$ \\ José Guilherme Mourão Dantas² \\ André Cezar Zingano ${ }^{3}$
}

\section{Resumo}

O uso de velocidade de onda sônica tem ganhado espaço na estimativa de resistência à compressão uniaxial (UCS) por ser um método mais barato, rápido e não destrutivo em comparação com o método tradicional. No entanto, por ser uma correlação empírica, as equações de correlação geradas variam muito quanto a precisão e necessitam serem aferidas para cada nova localidade geológica estudada. Com o objetivo de alcançar correlações mais precisas, esse artigo utiliza um maior critério de seleção das amostras. Arenitos e conglomerados criteriosamente selecionados resultaram em uma forte correlação com $R^{2}=0,99$. Dessa forma, se constatou que quanto mais específica for a litologia selecionada, melhor é a correlação obtida.

Palavras-chave: UCS; Pundit; Mecânica das rochas; Velocidade de onda sônica.

\section{CORRELATION EQUATION WITH EMPHASIS ON LITHOLOGY FOR UNIAXIAL COMPRESSIVE STRENGTH ESTIMATIVE BY THE USE OF PUNDIT}

\section{Abstract}

The use of sonic travel time has gained space in the estimative of uniaxial compressive strength (UCS) for being a cheaper method, faster and non-destructive in comparison with the traditional method. Nonetheless, for being an empirical correlation, the generated equations have much oscillation in its precision and need to be gauged for each new geological site. By using a strict criteria in the selection for the rock samples, this paper aims to reach correlations with a higher precision. Sandstones and conglomerates carefully selected resulted in a strong correlation with an $R^{2}=0,99$. Therefore, it was found that higher specificity in the selected lithology imply in a better correlation obtained.

Keywords: UCS; Pundit; Rock Mechanic; Sonic travel time.

1 Engenheiro de Minas, Mestrando, Departamento de Engenharia de Minas, UFRGS, Porto Alegre, RS, Brasil.

2 Engenheiro Geológico, Mestrando, Departamento de Engenharia de Minas, UFRGS, Porto Alegre, RS, Brasil.

3 Engenheiro de Minas, PhD, Professor/Pesquisador, Departamento de Engenharia de Minas, UFRGS, Porto Alegre, RS, Brasil. 


\section{INTRODUÇÃO}

Pare se obter uma classificação geomecânica completa para mineração ou engenharia geotécnica, é necessária uma extensa coleta de dados contendo propriedades e características das rochas. No entanto, devido a restrições econômicas, impossibilidade de amostragem, técnicas e de tempo, o trabalho de levantamento de dados deve ser objetivo, se atendo aos parâmetros de interesse. Dessa forma, métodos alternativos que forneçam estimativas geomecânicas baseadas em correlações empíricas ou teóricas são de grande ajuda (SHERAZ, 2014) [1].

Dentre os parâmetros procurados, um dos mais importantes é a resistência a compressão uniaxial (UCS), a qual representa uma propriedade chave para se determinar a estabilidade de obras de engenharia em rocha. A determinação da UCS para diferentes rochas em laboratório é uma prática comum e referência por fornecer um dado padronizado e confiável quanto a resistência da rocha. Todavia, esses métodos são caros, consumem tempo pois necessitam de uma preparação e ensaio com alta qualidade, precisão e sensibilidade (FENER et al, 2005) [2].

Por outro lado, testes indiretos como o ensaio de medição da velocidade de onda sônica para a estimativa de UCS das rochas necessitam de menor preparação das amostras, apresentam um tempo de ensaio menor e podem ser realizadas com facilidade se comparadas ao método tradicional. Diversos pesquisadores têm procurado correlações, as quais, com maior ou menor grau de precisão tem se mostrado satisfatórias para a estimativa de UCS. Segundo Butel (2014) [3] não há consenso entre os autores se cada localidade geológica necessita da sua própria correlação, ou se poderia ser feita por litologia. Sendo essas as duas vertentes de pensamento. Os autores procuram fazer correlações para as suas regiões e ao mesmo tempo dar ênfase nas litologias.

No Brasil, poucos estudos foram realizados nessa área (por exemplo GONZATTI, 2007) [4]. Dessa forma, esse estudo visa estabelecer uma correlação entre velocidade de onda sônica e resistência à compressão uniaxial para as rochas da localidade do sul do Brasil. Os ensaios serão empregados para amostras de diferentes litologias previamente selecionadas, buscando assim, diferentes equações de acordo com o tipo de rocha. As amostras analisadas foram restritas a arenitos e conglomerado provenientes de uma mina polimetálica localizada em Caçapava do Sul, no estado Rio Grande do Sul, Brasil.

\subsection{Geologia local:}

As amostras utilizadas para a pesquisa são oriundas do município de Caçapava do Sul, porção centro-sul do Estado do Rio Grande do Sul. Mais especificamente do distrito mineiro conhecido como Minas do Camaquã. Esta área está associada a evolução crustal da Bacia do Camaquã, datada do paleozoico, apresenta mineralizações sulfetadas de $\mathrm{Cu}, \mathrm{Pb}, \mathrm{Zn}$.

De acordo com PAIM e LOPES (2000) [5], o distrito mineiro das Minas de Camaquã está inserido no contexto evolutivo da Bacia do Camaquã. Os quais registram os episódios Vulcano-sedimentares. Esta sequência está representada por arenitos, conglomerados, arenitos conglomeráticos e ritmitos pelíticos

\subsection{Estimativa de resistência de rochas por métodos indiretos}


Comparativamente a esse método, o uso de velocidade ultrassônica tem se mostrado, recentemente, como uma opção válida para estimativa de UCS, considerando que é um teste não destrutivo, relativamente barato, rápido e fácil de ser realizado.

Os métodos indiretos utilizam propriedades físicas da rocha - como velocidade de onda sônica, densidade, resistividade, etc - para inferir medidas que normalmente são feitas diretamente. Diversos pesquisadores se aprofundaram nesses métodos, pois estes apresentam como característica principal a praticidade e viabilidade econômica. Tais métodos e suas tecnologias relacionadas foram sendo desenvolvidos principalmente junto a indústria do petróleo, a qual consegue ter conhecimento do cenário em subsuperfície sem precisar necessariamente de amostras físicas. (HOFFMAN, 1982) [6]

No que consiste a aplicação em geomecânica, os métodos geofísicos têm contribuído na identificação de fraturas, suas características e orientação, e também na estimativa da resistência à compressão uniaxial. Embora inúmeros pesquisadores tenham desenvolvido correlações entre propriedades físicas e UCS, não se tem conhecimento de haver uma correlação direta entre elas, dessa forma, todas as correlações existentes são empíricas.

Com base na boa correlação, diversos modelos empíricos foram desenvolvidos, todos com ajuste de uma equação a nuvem de pontos obtidas com o experimento sendo escolhida a linha de tendência que mais ajustasse a propriedade medida à resistência a compressão da rocha (SHARMA et al., 2010) [7]. Dentre os principais modelos criados três propriedades foram as mais exploradas: velocidade de onda $P$ $\left(\mathrm{V}_{\mathrm{p}}\right)$, porosidade $(\phi)$, módulo compressional $\left(\mathrm{M}=\rho \mathrm{V}^{2}\right)$. Vernik et al., (1993) [8] e Edlmann et al., (1998) [9] são exemplos de pesquisadores que desenvolveram correlações entre porosidade e UCS, com aplicação voltada a poços de petróleo. Com relação ao módulo compressional, Moos et al., (1999) [10] e Inoue e Ohomi (1981) [11] realizaram trabalhos obtendo correlações para conglomerados e anfibolitos, respectivamente. Já a velocidade de onda $P$, é a que tem sido mais explorada, apresentando tanto trabalhos em laboratório quanto em campo. Dezenas de pesquisadores têm procurado correlações para os tipos de rochas da sua região, obtendo correlações logarítmicas, exponenciais e lineares como, Freyburg (1972) [12], Militzer (1973) [13], McNally (1987) [14], Goktan (1988) [15], Grasso et al. (1992) [16], Lal (1999) [17], Tugrul e Zarif (1999) [18], Kahraman (2001) [19], Horsrud (2001) [20], Yasar and Erdogan (2004) [21], Entwisle et al. (2005) [22], Unpublished: Chang et al. (2006), Unpublished: Chang et al. (2006), Chary et al. (2006) [23], Oyler et al. (2007) [24], Sharma and Singh (2008) [25], Yagiz (2011) [26], Kurtulus et al. (2011) [27], Hakan and Derya (2012) [28], Azimian (2013) [29], Sheraz, (2014) [1], Butel (2014) [3].

Tabela 1: Equações que correlacionam velocidade de onda P com UCS.

\begin{tabular}{ll}
\hline Referência & Correlação empírica \\
\hline Freyburg (1972) & UCS $=35 \mathrm{Vp}-31,5$ \\
\hline Militzer (1973) & UCS $=(7682 / \Delta t)^{1.82} / 145$ \\
\hline Ohomi (1981) & UCS $=\mathrm{k} \rho \mathrm{Vp} 2+31.18$ \\
\hline McNally (1987) & UCS $=8,27 \mathrm{exp}(-0,036 \Delta \mathrm{t})$ \\
\hline Goktan (1988) & UCS $=36 \mathrm{Vp}-31,2$ \\
\hline Moos et al., (1999) & UCS $=1.745 \times 10^{-9} \times \rho \mathrm{Vp}^{2}-21$ \\
\hline Tugrul e Zarif (1999) & UCS $=35,54 \mathrm{Vp}-55$ \\
\hline
\end{tabular}




\begin{tabular}{|c|c|}
\hline Kahraman (2001) & UCS $=9.95 \mathrm{Vp}^{1.21}$ \\
\hline Horsrud (2001) & $\mathrm{UCS}=0.77(304.8 / \Delta t)^{2.93}$ \\
\hline Yasar e Erdogan (2004) & UCS $=(V p-2,0195) / 0,032$ \\
\hline Entwisle et al; (2005) & $U C S=0.783 e^{0.882 V p}$ \\
\hline Entwisle et al; (2005) & UCS $=0.292 \mathrm{Vp}^{4.79}$ \\
\hline Unpublished: Chang et al., (2006) & $\mathrm{UCS}=1.4138 \times 10^{7}\left(\Delta \mathrm{t}^{-3}\right)$ \\
\hline Chary et al; (2006) & $U C S=156,4 V p-692.41$ \\
\hline Chary et al; (2006) & $U C S=14,4 \mathrm{Vp}-24.856$ \\
\hline Mark et al., (2007) & $U C S=22,25 x \exp (-0,054 \Delta t)$ \\
\hline Sharma e Singh (2008) & $U C S=64,2 \mathrm{Vp}-117.99$ \\
\hline Yagiz (2011) & $U C S=49.4 \mathrm{Vp}-167$ \\
\hline Yagiz (2011) & $\mathrm{UCS}=0.258 \mathrm{Vp}^{3.543}$ \\
\hline Kurtulus et al; (2011) & $\mathrm{UCS}=67,5 \mathrm{Vp}-245.13$ \\
\hline Kurtulus et al; (2011) & $\mathrm{UCS}=18,8 \mathrm{Vp}-71.04$ \\
\hline Jabbar et al, (2011) & $\mathrm{UCS}=5363,64 \mathrm{Vp}$ \\
\hline Hakan e Derya (2012) & $U C S=14 \ln (V p / n)-96$ \\
\hline Hakan e Derya (2012) & $U C S=15 \ln (V p / n)-73$ \\
\hline Hakan e Derya (2012) & $U C S=0.0009(\mathrm{Vp} / \mathrm{n})+38$ \\
\hline Azimian (2013) & UCS $=0,026 \mathrm{Vp}-20,207$ \\
\hline Sheraz, (2014) & $U C S=218.8 V p-1413$ \\
\hline Butel et al; (2014) & $\mathrm{UCS}=1,11 \mathrm{e}^{0,9 \mathrm{~V} p}$ \\
\hline Freitas e Dantas (2017) & UCS $=4,0966 \mathrm{e}^{0,7394 x}$ \\
\hline
\end{tabular}

\subsection{Relação empírica entre propriedades físicas e a resistência da rocha}

A resistência a compressão uniaxial da rocha depende diretamente de suas propriedades como, porosidade, presença de água, absorção de água, tipo de mineral, densidade. Dentre essas, a porosidade e a velocidade de onda $P$ são as preferidas para se estimar a resistência (TUGRUL E ZARIFF, 1999) [30]. No entanto, tanto a velocidade de onda $\mathrm{P}$ quanto a porosidade só apresentam relações matemáticas entre si e com outras propriedades, mas não com o UCS, sendo essas relações empíricas, e geradas a partir da análise do comportamento dos resultados. Embora todas essas propriedades físicas sejam acessíveis e de fácil medição, ainda não há uma equação de correlacione os diversos fatores que influenciam na medição da resistência.

As equações 1,2 e 3 mostram a as relações matemáticas entre as propriedades passiveis de medição.

$$
\varphi=\frac{\left(\rho_{m}-\rho_{b}\right)}{\left(\rho_{m}-\rho_{f}\right)}
$$

Onde $\varphi$ é a porosidade e $\rho_{m}, \rho_{b}$ e $\rho_{f}$ são respectivamente a densidade da matriz, densidade da formação e densidade do fluido.

$$
v_{p}=(1-\varphi)^{2} v_{p s}+\varphi v_{p f}
$$

A porosidade é determinada por uma relação entre a velocidade de onda- $P$ total $\left(v_{p}\right)$, relacionado a velocidade da fase sólida $\left(v_{p s}\right)$ e de fase com fluido $\left(v_{p f}\right)$.

$$
v_{p s}=\left[\frac{K+4 / 3 \mu}{\rho_{s}}\right]^{1 / 2}
$$



da matriz.

Onde $\mathrm{K}$ é densidade volumétrica, $\mu$ é o módulo de rigidez e $\rho_{s}$ é a densidade

\subsection{Diferença de comportamento em função da litologia}

De acordo com Soroush e Qutob (2011) [31] a relação entre velocidade de onda $P$ e UCS está intimamente ligado ao tipo de rocha analisada (Figura 1). Isso se deve à variação no modo como a onda vai ser transmitida de acordo com a formação geológica, se é clástica, vulcânica ou metamórfica, os quais se somam aos demais fatores de porosidade, saturação e densidade.

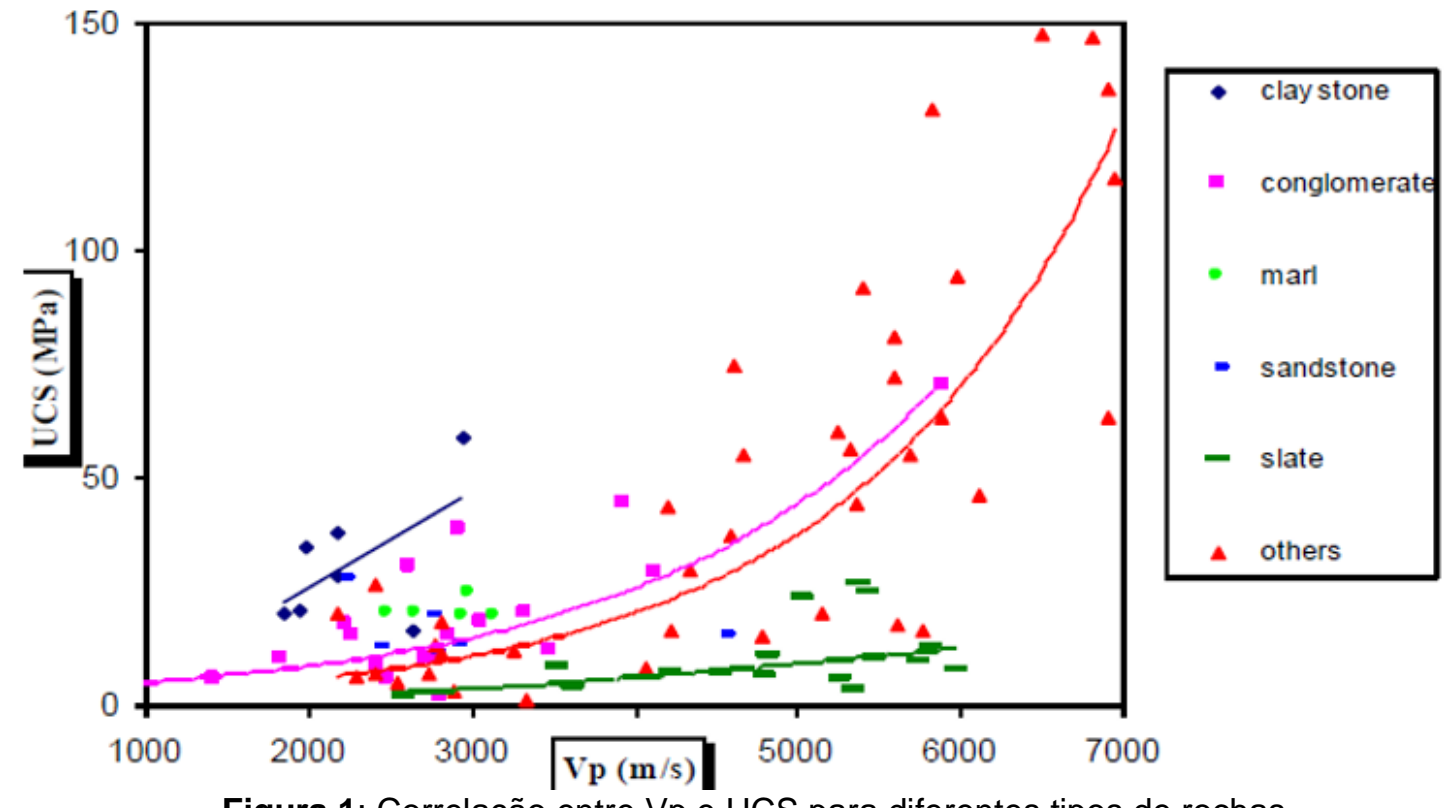

Figura 1: Correlação entre Vp e UCS para diferentes tipos de rochas.

\section{MATERIAIS E MÉTODOS}

\subsection{Ensaio de Resistência a Compressão Uniaxial:}

Resistência a compressão uniaxial é a capacidade que uma rocha tem de suportar tensões compressivas em um único eixo. Tal medida, foi normatizada pela American Society for Testing and Materials (ASTM) e International Society for Rock Mechanics (ISRM). As referências normativas utilizadas neste ensaio foram: ASTM D 4543-85 e ABNT NBR 15845:2010.'

O ensaio é realizado em laboratório utilizando uma prensa hidráulica controlada que aumenta gradativamente a carga sobre a amostra até rompê-la (Figura 2). Embora a padronização do ensaio seja importante por fornecer um dado que traduz a qualidade da rocha, ela também gera limitações em relação às rochas que podem ser ensaiadas devidos as dimensões dos corpos de provas, os quais precisam ter uma razão mínima de altura-diâmetro de 2:1. Isso exige que a rocha seja minimamente competente para suportar a preparação, o que geralmente é um problema em formações fraturadas, intemperizadas ou em camadas finas (AZIMIAN, 2013) [29]. 


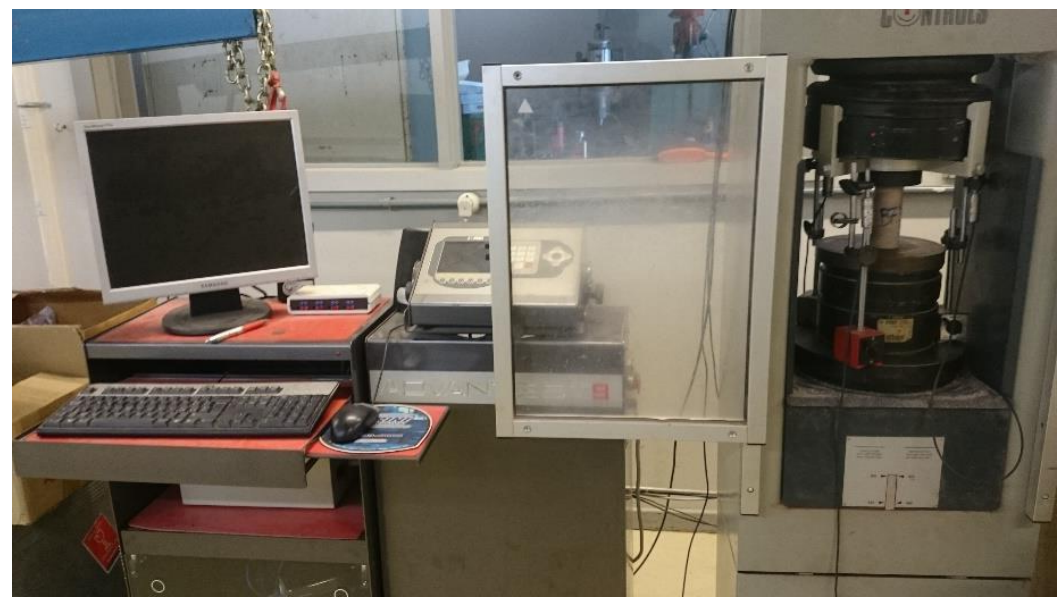

Figura 2: Prensa hidráulico (Control Advanced 9), utilizada nos ensaios.

Apesar da consagração do método, a obtenção de resultados por esse ensaio também apresenta pontos negativos por ser cara, além de ser um teste destrutivo (GOKCEOGLU and ZORLU, 2004 [32]; BAYKASOGLU et al. 2008 [33]). Dessa forma, métodos que forneçam resultados similares e que ainda possam contrapor os pontos negativos do ensaio atual são vistos como uma boa alternativa. Dentro desse contexto, diversos métodos indiretos têm mostrado vantagens ao estimar a resistência a compressão uniaxial em rochas que o método atual falha, além de possuírem características vantajosas como a praticidade, custo e preservação da amostra.

\subsection{Ensaio de Determinação de Velocidade de Ultrassônica:}

A velocidade de propagação de onda ultrassônica é determinada com o uso do Pundit (Portable Ultrasonic Nondestructive Index Tester), os ensaios são simples e com baixo custo, necessitando somente uma preparação das superfícies das amostras que serão ensaiadas (Figura 3). Esse método de ensaio descreve as medições laboratoriais das velocidades de compressão (onda p) e cisalhamento (onda s) da rocha.

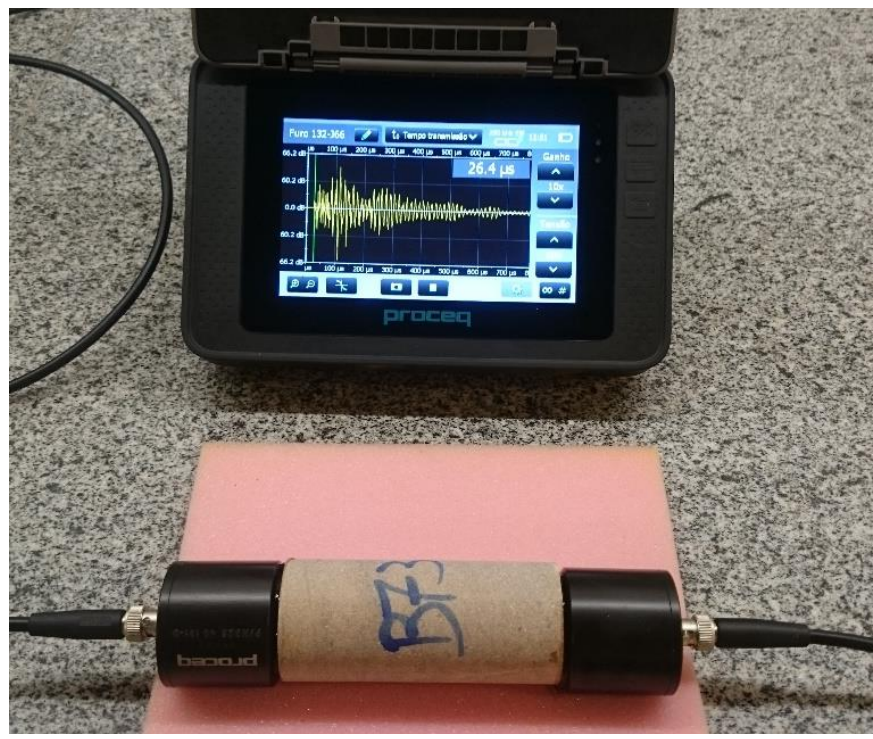

Figura 3: Pundit utilizado nos ensaios. 
Essas velocidades podem ser influenciadas pela porosidade, grau de absorção de água, composição mineralógica das rochas e descontinuidades. A velocidade de propagação de onda é dependente das propriedades elásticas do material. Quanto maior for a resistência mecânica, maior será a coesão e maior será a velocidade de propagação. Também é influenciada pela densidade dos minerais constituintes da rocha.

A norma técnica referente a esse ensaio é a ASTM D 2845-08. No ensaio foi utilizado o Pundit PL 200 da Proceq, com transdutores de $54 \mathrm{kHz}$ e uma frequência de $250 \mathrm{kHz}$. Para a medição de velocidade nas amostras é necessário o uso de gel acoplador com o intuito de possibilitar a passagem da onda de compressão, do transdutor para a amostra e vice-versa.

\subsection{Preparação das Amostras:}

As amostras a serem analisadas necessitam satisfazer as implicações dos dois ensaios, primeiramente o de medição da velocidade de onda, método não destrutivo, e posteriormente o ensaio de compressão uniaxial, destrutivo. Um levantamento, das principais exigências das normas acima citado, foi realizado e citados abaixo:

- Apresentam diâmetro "NX", aproximadamente 54,00 mm

- Corpos cilíndricos tem que apresentarem relação de dimensões entre base e altura de 2:1 até 2,5:1;

- As faces das amostras devem ser, obrigatoriamente, plana e paralelas entre si (com uma tolerância máxima de 0,02 mm);

- Não pode ter aplicações de aditivos (colas, massa) para tratamento nas faces das amostras;

- O comprimento de onda tem que ser $3 \times$ o tamanho médio dos grãos. Para diâmetro "NX", com frequência de 250 kHz ou 500 kHz, o tamanho máximo dos grãos seria de $5 \mathrm{~mm}$ ou $2,33 \mathrm{~mm}$, respectivamente.

Após a preparação das amostras, as mesmas são catalogas e recebem um código para identificação. Detalhes como massa, comprimento, diâmetro são anotados em fichas descritivas. As mesmas são fotografadas e inseridas no banco de dados.

\subsection{Seleção das amostras}

As amostras foram selecionadas para que só se analisassem rochas da mesma litologia e que fossem similares. Tal critério foi adotado pois se notou em experimentos que a análise conjunta de diversos tipos de rocha não acarretava em boas correlações.

A seleção das rochas analisadas - arenitos e conglomerados - foi feita de forma criteriosa observando-se os seguintes itens:

- Granulometria

- Grau de selecionamento dos grãos (bem selecionado ou mal selecionado)

- Grau de alteração

\section{RESULTADOS E DISCUSSÃO}

Foram ensaiadas 32 amostras de arenito, sendo dividas em arenitos bem selecionados e mal selecionados. Também foram ensaiadas 12 amostras de 
conglomerado. Não apresentavam sinais de alteração. Todas foram preparadas de forma similar obtendo uma padronização com amostras com relação altura/diâmetro igual a 2:1.

Os resultados obtidos na forma de dispersão de pontos (Figura 4) foram testados quanto o melhor ajuste de linha de tendência (Tabela 2Tabela 1). A juste na forma de potência trouxe uma maior correlação para os dados obtidos.

Tabela 2: Teste de ajuste de correlação

\begin{tabular}{llc}
\hline Correlação & Equação & Coeficiente de correlação \\
\hline Linear & UCS $=28,031 \mathrm{Vp}-44,884$ & $\mathrm{R}^{2}=0,9854$ \\
\hline Exponencial & UCS $=4,0966 \mathrm{e}^{0,7394 \mathrm{~V}}$ & $\mathrm{R}^{2}=0,9935$ \\
\hline Logarítmo & $\mathrm{UCS}=81,618 \ln (\mathrm{Vp})-49,859$ & $\mathrm{R}^{2}=0,9644$ \\
\hline Potência & $\mathrm{UCS}=3,5015 \mathrm{Vp} \mathrm{p}^{2,178}$ & $\mathrm{R}^{2}=0,995$ \\
\hline
\end{tabular}

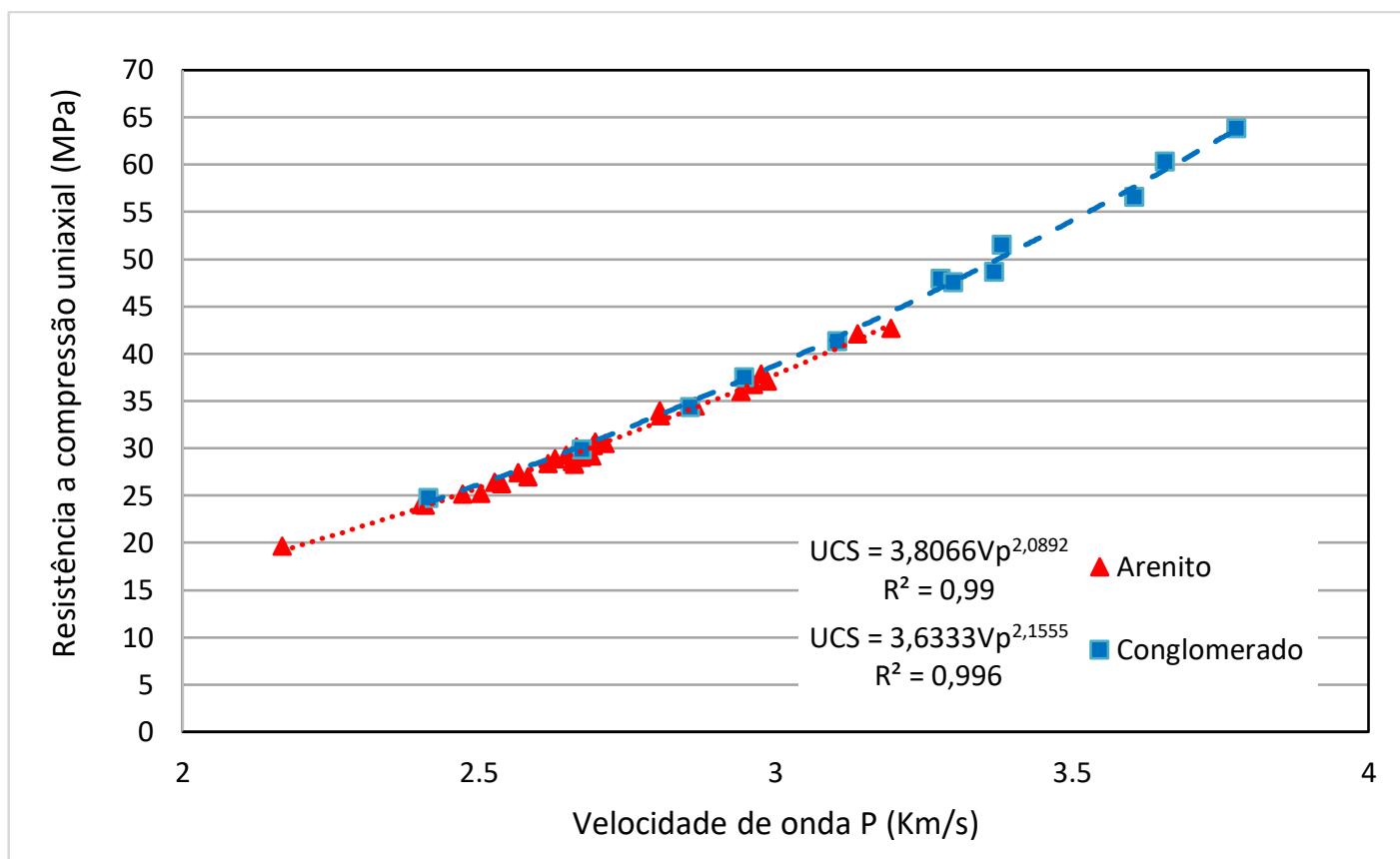

Figura 4: Correlação entre Vp e UCS para as amostras de conglomerado e arenito.

O resultado com uma correlação tão precisa com $R^{2}>0,99$ é atribuído principalmente a um maior critério para a seleção das rochas.

Embora se esperasse uma dispersão dos valores de forma diferente na comparação das litologias arenito e conglomerado, verificou-se que para a geologia em questão eles se comportam de forma similar (Figura 5). Também não foi verificado diferença entre as amostras de arenitos mal selecionados e bem selecionados. Dessa forma, todas as amostras podem ser analisadas, sem erro significativo de acordo com a equação obtida (Equação 4).

$$
U C S=3,5015 * \mathrm{Vp}^{2,178}
$$

(Equação 4) 


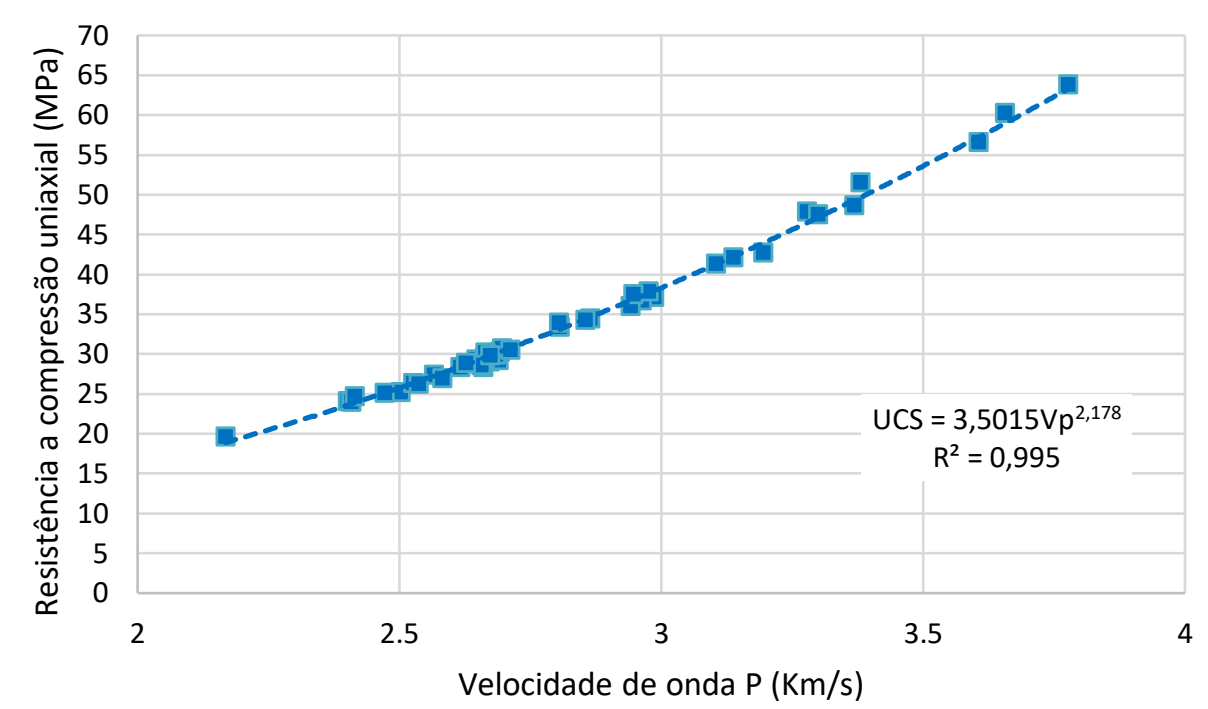

Figura 5: Correlação entre velocidade e propagação de onda e Resistência a compressão uniaxial para todas as amostras.

Em comparação como outros trabalhos, nos quais foram analisados arenitos, é muito nítida a semelhança de comportamento das correlações obtidas (Figura 6). Butel (2014) [3] e McNally (1987) [14] utilizam uma correlação na forma exponencial; Chang et al (2006), assim como esse trabalho, adota uma correlação na forma de potência; já Chary et al (2006) trabalha com dois conjuntos de dados separados, os quais se ajustam melhor em uma correlação linear, no entanto se fossem analisados juntos resultariam em uma correlação na forma exponencial ou de potência. Desse modo, fica evidente um padrão de comportamento na análise litológica desse tipo de correlação.

Com relação a translação do gráfico obtido em comparação com o de outros autores, não há uma explicação imediata, podendo-se atribuir a um conjunto de fatores. É possível que seja atribuído a uma característica local, como alguns autores defendem (McNally, 1987 [14]; Sharma and Singh, 2008 [25]). Ou pode ser atribuído ao método de ensaio, pois McNally (1987) [14], Chang et al (2006) e Butel (2014) [3] obtiveram seus valores de velocidade de onda P por medidas in situ, utilizando perfilhadores sônicos. Estes, embora apresentem o mesmo princípio de funcionamento do PUNDIT, atuam em meio líquido no furo de sondagem, podendo explicar a diferença nas respostas.

Embora Soroush e Qutob (2011) [31] não tenham desenvolvido uma equação devido ao baixo número de dados (Figura 1), suas medidas para arenito também foram feitas com PUNDIT, e da mesma forma estão transladadas para cima como o deste trabalho, o que pode indicar que tal mudança seja atribuída ao tipo de método utilizado. A diferença dá-se no fato de o PUNDIT medir amostras secas e em laboratório, enquanto o perfilador sônico mede estratos in situ e imersos em água, o que aumenta a velocidade registrada em comparação ao ar (WELLS, 1990 [34]). 


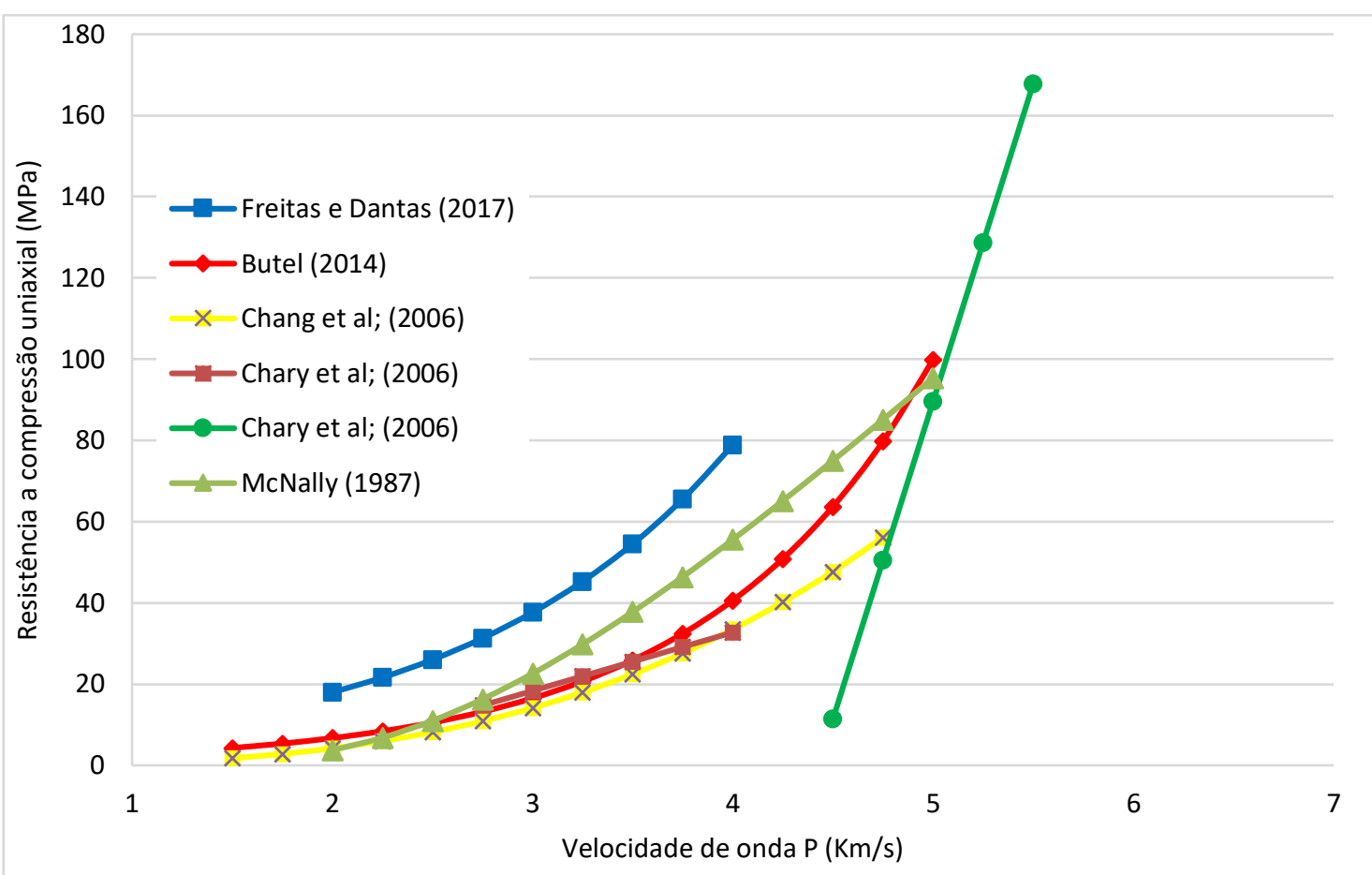

Figura 6: Correlações obtidas para arenitos por diferentes autores.

\section{CONCLUSÃO}

A relação entre velocidade de onda $P$ e resistência a compressão uniaxial verificou-se de forma positiva para as rochas da região estudada, onde obteve-se uma equação que permite estimar a resistência compressão da rocha somente com a medida da sua velocidade.

Ainda que sejam classificadas como correlações empíricas, verificou-se que um maior critério na seleção das amostras para construção da curva de correlação pode levar a resultados mais precisos. Dessa forma tal método pode ser aplicado em estimativas de resistência com maior exatidão ao se separar por litologias. No entanto, não se pode generalizar resultados para regiões inteiras, devendo sempre ser averiguado o comportamento das rochas em estudo para ver se estas se aplicam as correlações elaboradas.

Espera-se testar de forma criteriosa também outras litologias a fim de se estabelecer novas correlações e aumentar a confiança da estimativa de UCS pelo uso de Pundit.

\section{Agradecimentos}

Os autores agradecem a CAPES pela bolsa de estudo, agradecem a Fundação Luiz Englert pelo financiamento do projeto, e à Votorantim Metais por ceder amostras para realização dessa pesquisa.

\section{REFERÊNCIAS}


1 AM Sheraz, MZ Emad, M Shahzad, SM Arshad. Relation between uniaxial compressive strength, point load index and sonic wave velocity for dolerite. Pakistan Journal of Science Vol. 66 No. 1. 2014; 66 (1), 60-66

2 Fener M, Kahraman S, Bilgil A, Gunayadin O. A comparative evaluation of indirect methods to estimate the compressive strength of rocks. Rock Mechanics and Rock Engineering. 2005 ; 38(4): 329-343 ().

3 Butel N, Hossack A, Kizil MS. Prediction of in situ rock strength using sonic velocity. 14th Coal Operators' Conference. 2014; 89-102.

4 Gonzatti, C. (2007). Proposta para a estimativa da resistência in situ de camadas de carvão com a utilização de geofísica. Escola de Engenharia de São Carlos da Universidade de São Paulo (Tese de Doutorado). 274p.

5 Paim PSG, Lopes RC. Geologia da Região das Minas do Camaquã. In: Rochi, L.H., \& Lobato, A.O.C.(Eds), Minas do Camaquã, um estudo muldidisciplinar. São Leopoldo, Unisinos. 2000; 111-132.

6 Hoffman GL, Jordan GR, Wallis GR. Geophysical borehole logging handbook for coal exploration. The Coal Mining Research Centre, Edmonton, Alberta, Canada, 1982. 270p.

7 Sharma MSR, O'regan M, Baxter CDP, Moran K, Vaziri H, Narayanasamy R. Empirical relationship between strength and geophysical properties for weakly cemented formations. Journal of Petroleum Science and Engineering. 2010; 72, 134-142.

8 Vernik L, Bruno M, Bovberg C. Empirical relations between compressive strength and porosity of siliciclastic rocks. Int. J. Rock Mech. Min. Sci. Geomech. Abstr. 1993; 30, 677-680.

9 Edlmann K, Somerville JM, Smart BGD, Hamilton SA, Crawford BR. Predicting Rock Mechanical Properties from Wireline Porosities. Paper presented at the SPE/ISRM Eurock '98 held in Trondheim, Norway, 8-10 July 1998, SPE 47344.

10 Moos D, Zoback MD, Bailey L. Feasibility Study of the Stability of Openhole Multilaterals, Cook Inlet, Alaska. 999 SPE Mid-Continent Operations Symposium held in Oklahoma City, Oklahoma, 28-31 March 1999, SPE 52186.

11 Inoue M, Ohomi M. Relation between Uniaxial Compressive Strength and Elastic Wave Velocity of soft rock. Proceedings of the International Symposium on Weak Rock, Tokyo, 1981; 9-13.

12 Freyburg E. Der Untere und mittlere Buntsandstein SW-Thuringen in seinen gesteinstechnicschen Eigenschaften. Ber. Dtsch. Ges. Geol. Wiss., A; Berlin. 1972; 176, 911-919.

13 Militzer, H., Stoll, R., 1973. Einige Beitrageder geophysics zur primadatenerfassung im Bergbau, Neue Bergbautechnik. Lipzig 3, 21-25.

14 McNally, GH. Estimation of coal measures rock strength using sonic and neutron logs. Geoexploration. 1987; 24, 381-395.

15 Goktan RM. A theoretical and application analyses of the rock diggability (in Turkish), Ph.D. Thesis, 1988, ITU Institute of Appl. Sciences, p. 108.

16 Grasso P, Xu S, Mahtab A. Problems and promises of index testing of rocks. Proceedings of the 33rd U.S. Symposium on Rock Mechanics, Balkema, Rotterdam, 1992; 879-888.

17 Lal M. Shale stability: drilling fluid interaction and shale strength. SPE Latin American and Caribbean Petroleum Engi-neering Conference held in Caracas, Venezuela. 1999

18 Turgrul A, Zarif IH. Correlation of mineralogical and textural characteristics with engineering properties of selected granitic rocks from Turkey. Eng Geol. 1999; 51:303317

19 Kahraman S. Evaluation of simple methods for assessing the Uniaxial Compressive Strength of rock. International Journal of Rock Mechanics and Mining Sciences. 2001; 38(7): 981-994.

20 Horsrud P. Estimating mechanical properties of shale from empirical correlations. SPE Drill. Complet. 2001; 16, 68-73. 
21 Yasar E, Erdogan Y. Correlating sound velocity with density, compressive strength and Young's modulus of carbonate rocks. International Journal of Rock Mechanics and Mining Sciences. 2004; v. 41, p. 871-875.

22 Entwisle DC, Hobbs PRN, Jones LD, Gunn D, Raines MG. The Relationships between Effective Porosity, Uniaxial Compressive Strength and Sonic Velocity of intact Borrowdale Volcanic Group core samples from Sellafield. Geotechnical and Geological Engineering. 2005; 23: 793-809.

23 Chary K.B., L.P. Sarma, K.J. Prasanna Lakshmi, N.A. Vijaya kumar, V. Naga Lakshmi and M.V.M.S. Rao. Evaluation of engineering properties of rock using Ultrasonic Pulse Velocity and Uniaxial Compressive Strength. Proceedings National Seminar on NonDestructive Evaluation. 2006; 379-385.

24 Oyler, D C, Mark, C, Molinda, G. Correlation of sonic travel time to the uniaxial compressive strength of U.S. coal measure rocks, Proceedings of the 27th International Ground Control in Mining Conference, Morgantown, WV. 2008. pp 338-346.

25 Sharma P.K. and T.N. Singh. A correlation between P-Wave velocity, impact strength index, slake durability and Uniaxial Compressive Strength. Bull Eng. Environ. 2008; 67: 17-22.

26 Yagiz S. P-Wave velocity test for assessment of geotechnical properties of some rock materials. Bull Mater Science. 2011; 34(4): 947-953.

27 Kurtulus C, Bokkurt A, Endes H. Physical and Mechanical Properties of Serpentinized Ultrabasic Rocks in NW Turkey. Pure and applied Geophysics. 2011; 169(7):1-11

28 Hakan E, Darya K. Multicriteria decision making analysis based methodology for predicting carbonate rocks Uniaxial Compressive Strength. Earth Sciences Research Journal. 2012; 16: 65-74.

29 Azimian A, Ajalloeian R, Fatehi L. An empirical correlation of uniaxial compressive strength with $p$-wave velocity and point load strength index on marly rocks using statistical method. Geotech. Geol. Eng. 2014; 32:205-214

30 Tugrul A, Zarif IH. Correlation of mineralogical and textural characteristics with engineering properties of selected granitic rocks from Turkey. Engineering Geology. 1999; 51: 303-317

31 Soroush $\mathrm{H}$, Qutob $\mathrm{H}$. Evaluation of rock properties using ultrasonic pulse technique ad correlating static to dynamic elastic constants. GEOIndia. 2011.

32 Gokceoglu, C., Zorlu, K. (2004). A fuzzy model to predict the uniaxial compressive strength and the modulus of elasticity of a problematic rock. Engineering Applications of Artificial Intelligent, 17(1):61-72.

33 Baykasog lu A, Gu 'llu "H, C, anakc, H, O "zbakır L (2008) Predict-ing of compressive and tensile strength of limestone via genetic programming. Expert Syst Appl 35:111-12.

34 Wells PE. Porosities and seismic velocities of mudstones from Wairarapa and oil wells of North Island, New Zealand, and their use in determining burial history, New Zealand Journal of Geology and Geophysics. 1990; 33:1, 29-39 